\title{
Análisis de la Implementación del Teletrabajo durante la pandemia del COVID-19
}

doi: 10.33264/rpa.202101-07

Fernando Cortes Tello, Carrera de Enfermería Coquimbo, Universidad Central de Chile Diego Silva Jiménez, Facultad de Ciencias de la Salud, Universidad Central de Chile. Daniela Muñoz Marín, Carrera de enfermería Coquimbo, Universidad Central de Chile Romina Lizondo Valencia, Carrera de enfermería, Universidad Central de Chile

\section{Resumen}

El teletrabajo es cuando un empleado cumple sus funciones regulares desde su hogar u otro lugar que no sean las dependencias de su organización, esta modalidad laboral tiende a aumentar en épocas de crisis, como en la actual pandemia del COVID-19. El objetivo de esta investigación fue analizar la implementación del teletrabajo en la salud de los trabajadores y sus familias durante el confinamiento por covi-19. La metodología utilizada fue la revisión narrativa para analizar la literatura disponible sobre el teletrabajo y su impacto en la Salud de los trabajadores que desempeñan sus labores en esta modalidad y la conciliación de la vida laboral y familiar, en un contexto de Pandemia. Los resultados obtenidos fueron que el teletrabajo genera riesgos para la salud mental y física de los trabajadores, afectando su vida familiar y sus espacios de ocio. Persisten factores de riesgo propios de cualquier lugar de trabajo como los ergonómicos y psicosociales, pero existen factores de riesgo por estudiar en un ámbito domiciliario. Como conclusiones, el teletrabajo debe ser abordado de manera urgente por parte del estado, fortaleciendo la legislación laboral pertinente, abordando la promoción de la salud, así como la prevención de los riesgos e implementando medidas que permitan mitigar los efectos del teletrabajo, siguiendo recomendaciones elaboradas por organismos internacionales, pero por sobre todo desarrollando sistemas de vigilancia epidemiológica que permitan evitar la subnotificación de casos de enfermedades y accidentes relacionados al teletrabajo, que requieren ser identificados, en atención a la mantención de esta modalidad.

Palabras clave: teletrabajo, salud ocupacional, pandemia, legislación laboral, vida familiar

\section{Abstract}

Teleworking is when an employee performs his regular functions from his home or another place other than the dependencies of his organization, this work modality tends to increase in times of crisis, such as in the current COVID-19 pandemic. The objective of this research was to analyze the implementation of telework in the health of workers and their families during confinement by covi-19. The methodology used was the narrative review to analyze the available literature on teleworking and its impact on the Health of workers who carry out their work in this modality and the reconciliation of work and family life, in a context of Pandemic. The results obtained were that telework generates risks for the mental and physical health of workers, 
affecting their family life and leisure spaces. Risk factors typical of any workplace persist, such as ergonomic and psychosocial ones, but there are risk factors to study in a home setting. As conclusions, telework must be urgently addressed by the state, strengthening the pertinent labor legislation, addressing health promotion, as well as risk prevention, and implementing measures to mitigate the effects of teleworking, following recommendations made by international organizations, but above all by developing epidemiological surveillance systems that make it possible to avoid the under-notification of cases of diseases and accidents related to telework, which need to be identified, in attention to the maintenance of this modality.

Keywords: telecommuting, occupational health, pandemic, labor law, family life

\section{Teletrabajo; Historia y definición}

La definición de teletrabajo planteada por Jack Nilles cumple 48 años y es "aquella forma de enviar el trabajo al trabajador, en lugar de enviar el trabajador al trabajo" (López, Pérez-Simon,Nagham-Ngwessitcheu \& Vázquez-Ubago, 2014,p.589), Sin embargo, esta nueva modalidad corre el riesgo de una mayor carga laboral para el trabajador ya que puede aumentar el estrés laboral que genera la interferencia familiar, siendo esta una arma de doble filo entre la conciliación de la vida familiar con la vida laboral, por lo que hay que tener cuidado en promocionar la igualdad de género en los trabajadores que desempeñan en dicha modalidad, para que esto funcione es la organización quien debe entregar el mobiliario necesario o apoyar económicamente a instalar el puesto de trabajo en el domicilio. En esta misma lógica las personas que realizan el teletrabajo están susceptibles a riesgos laborales, que aún no están completamente identificados, siendo el empleador el que debe entregar un puesto de (tele)trabajo libre de peligro, con las medidas de prevención como si estuviera en modalidad presencial. (López, Pérez-Simon,NaghamNgwessitcheu \& Vázquez-Ubago, 2014)

El 2002 la Unión Europea firmó el “Acuerdo Marco Europeo Sobre El Teletrabajo” que busca modernizar la organización del trabajo enfocados en el equilibrio necesario entre flexibilidad y seguridad, con énfasis en los siguientes puntos; los teletrabajadores tienen los mismos derechos que los trabajadores que se desempeñan en las instalaciones de la organización; el empleador debe realizar la adecuación del lugar de trabajo destinado para el teletrabajo, tales como, la instalación y mantenimiento de los distintos equipos utilizados para dicha labor; el empleador es responsable de la salud y de la seguridad profesional del teletrabajador ; y por ultimo dicha vigilancia requiere que el empleador tenga acceso al hogar del teletrabajador. (Rodríguez, 2007). 
El teletrabajo se puede definir a través de conceptos claves, el trabajo, la distancia y el uso intensivo de TIC. La persona trabaja fuera o alejada de las oficinas de la organización o empresa para la cual trabaja de manera computarizada, pudiendo ser estos últimos no solo computadores, sino que celulares, Tablet u otro dispositivo, ya sea personal o de la organización y en esta definición la relación contractual del trabajador no genera ninguna diferencia, dado que la persona puede estar contratada o que preste servicios de manera freelance (Osio,2010).

Desde 2014 hay referencia al teletrabajo en países de Latinoamérica, como los casos de Ecuador, Chile y Colombia, este último, donde se han podido identificar algunas condiciones para su funcionamiento y que tiene relación con las consiguientes leyes del trabajo y su marco normativo, como lo son, los riesgos laborales, la seguridad de la información, la protección de la información y de los datos sensibles, junto con aspectos de seguridad laboral dentro de hogar, en la medida telemática (Buitrago, 2020).

A pesar del aumento del desarrollo y auge del teletrabajo falta desarrollo de la legislación jurídico-legal, junto con la implementación de medidas de seguridad en el teletrabajo, esto es muy preocupante, debido a que en varios países aún no se reconoce el teletrabajo como actividad laboral contractual, esto se da debido a su enfoque hacia la flexibilidad y la inclusión de las personas con limitación de movilidad por discapacidad física y conciliación de la vida familiar. También hay desventajas en la aplicación de esta modalidad de trabajo remoto, que tiene directa relación con su salud, como el aislamiento, exceso de trabajo (adicción al trabajo) y otras problemáticas que pueden ser asociadas a riesgos psicosociales, que son muy difíciles de identificar en este tipo de trabajadores, a pesar de esto hay pocos estudios sobre dicha materia. (Bonilla, Plaza, De Cerquera \& Riaño-Casallas, 2014).

Independiente de las problemáticas y la falta de regulación asociadas al teletrabajo, que fueron planteadas con anterioridad, se puede encontrar puntos favorables a la implementación de este y se pueden asociar a diversos ámbitos, desde el punto de vista de la organización/empresa y familia, tales como ; disminución de costos, relacionados con mayor productividad, acceso y flexibilidad laboral, especialmente a personas con discapacidad, también se puede observar menor ausentismo y rotación laboral, asociado a una mayor flexibilidad horaria. Dentro del ámbito social - familiar, se puede rescatar, la posibilidad de mayor cercanía de los y las jefes de hogar en la crianza de sus hijos y en el ámbito tecnológico, un manejo más rápido de la información, aumento del uso e implementación de TICs, y finalmente un menor tráfico vehicular, lo que ayudaría en la reducción de la contaminación y congestión vehicular. (Buitrago, 2020; López, Pérez-Simon,Nagham-Ngwessitcheu \&VázquezUbago, 2014). 
El teletrabajo tiende a aumentar cuando hay crisis en los países o el mundo, ejemplo de ello, es el gran auge de distintas investigaciones a nivel de teletrabajo en relación con la crisis subprime de Estados Unidos que fue originada por la burbuja inmobiliaria, en el año 2008 hasta el 2012, generando inestabilidad en los distintos mercados financieros, lo que desencadeno el aumento del teletrabajo como medida de flexibilidad laboral y económica (Silva, 2014).

En la actualidad la mayoría de las personas han visto interrumpida su vida cotidiana, debido a la pandemia del covid-19, siendo una de las más relevantes los confinamientos obligatorios, teniendo como consecuencia la aplicación forzada de un contexto de teletrabajo. (Lizondo, Silva, Arancibia, Cortes \& Muñoz, 2021)

Es en base a lo anteriormente expuesto que el objetivo de esta investigación fue analizar la implementación del teletrabajo en la salud de los trabajadores y sus familias durante el confinamiento por covid-19.

\section{Metodología}

La metodología utilizada fue la de revisión Narrativa, que "suele ser de mirada amplia, pero el sustento científico entregado es variable y dependerá del autor; suele transmitir también en mayor o menor grado, la experiencia de los autores" (Salinas, 2020.p.27), "la característica común en la revisión narrativa es que se revisa la literatura publicada, y ello implica que los materiales incluidos poseen cierto grado de permanencia" (Guirao, 2015.sp).

En base a ello se revisaron artículos científicos de distintas base de datos, Latindex, DOAJ, Scielo, Scopus, LILACs, además de documentos de oficiales de organismos internacionales y cuerpos legales vigentes, sobre teletrabajo, COVID-19, riesgos de salud y salud ocupacional, para lograr desarrollar un análisis sobre la implementación del teletrabajo en Pandemia, revisando la legislación existente para abordar una nueva condición laboral en contexto de emergencia sanitaria, y como los riesgos laborales se ven asociados al hogar, impactando la conciliación de la vida familiar y los nuevos requerimientos del mundo laboral. Por ello se analizan recomendaciones de organismos internacionales para su abordaje (Vera, 2009).

En este caso, las categorías para agrupar esta información fueron cuatro: Legislación Laboral referente al Teletrabajo, Riesgos para la Salud del Teletrabajo, Análisis epidemiológico relacionado al Teletrabajo y la Salud Ocupacional, y el Contexto del teletrabajo y conciliación con la vida familiar, estas fueron analizadas, desarrolladas en capa acápite y resumidas al final de los resultados. 


\section{Resultados}

A continuación, se desarrolla la revisión narrativa del teletrabajo en relación con cuatro categorías emergentes que son: Legislación Laboral referente al Teletrabajo, Riesgos para la Salud del Teletrabajo, Análisis epidemiológico relacionado al Teletrabajo y la Salud Ocupacional, y el Contexto del teletrabajo y conciliación con la vida familiar, para finalmente entregar algunas recomendaciones para su abordaje.

\section{a. Legislación Laboral referente al Teletrabajo:}

La legislación chilena establece en la Ley 21220, que modifica el código del trabajo en materia de trabajo a distancia, en su artículo 152 quáter $\mathrm{G}$, que el trabajo a distancia es aquel en el que el trabajador presta sus servicios, desde su domicilio u otro lugar distinto a las instalaciones de la empresa (Ley 21220, 2020). Concordantemente con lo anterior denomina el teletrabajo a aquel en los que "los servicios son prestados mediante la utilización de medios tecnológicos, informáticos o de telecomunicaciones o si tales servicios deben reportarse mediante estos medios". (Ley 21220, 2020, p1).

Por otro lado, el teletrabajo ha sido definido como el uso de tecnologías de la información y las comunicaciones -como teléfonos inteligentes, tabletas, computadoras portátiles y de escritorio- para trabajar fuera de las instalaciones del empleador (Eurofound y OIT, 2019). En otras palabras, “el teletrabajo conlleva un trabajo realizado con la ayuda de las TIC, fuera de las instalaciones del empleador" (OIT, 2020, P1).

En este sentido la ley establece, además que las partes podrán pactar la distribución de la jornada laboral, respetando la jornada diaria y semanal. También establece que el empleador debe respetar el derecho a desconexión, garantizando periodos de tiempo en que no se obligará al trabajador a responder comunicaciones o solicitudes, respetando la legislación pertinente (Ley 21220, 2020).

Por otra parte, la Ley plantea en su artículo 152 quáter L, que los equipos, las herramientas y los materiales, incluyendo elementos de protección personal "deberán ser proporcionados por el empleador Igualmente, los costos de operación, funcionamiento, mantenimiento y reparación de equipos serán siempre de cargo del empleador" (Ley 21220, p2).

En el caso de las condiciones específicas de seguridad y salud, Decreto 18 de 2020 dictado el Ministerio del Trabajo y Previsión Social, establece en su artículo 2, que:

Los trabajadores que pacten con sus empleadores la modalidad de trabajo 
a distancia o teletrabajo, al inicio o durante la relación laboral, tendrán iguales derechos y obligaciones en materia de seguridad y salud en el trabajo que cualquier otro trabajador, salvo aquellas adecuaciones que deriven estrictamente de la naturaleza y características de la prestación convenida (Decreto 18, 2020, p1).

Por lo anterior estarán sujetos a todas las disposiciones de la Ley 16.744 y su respectiva legislación correspondiente en las materias específicas que se generen por la exposición a riesgos a causa o con ocasión del trabajo (Ley 16.744, 2017).

\section{b. Riesgos para la Salud del Teletrabajo:}

Algunos de los riesgos que el teletrabajo incrementa en la salud mental y física de los trabajadores son la sobrecarga o subcarga de trabajo, que se puede asociar a afectación de sus ritmos biológicos, generación de estrés, ansiedad, irritabilidad, estados depresivos, temor y aburrimiento, sensación de aislamiento, ambigüedad o conflicto de rol, riesgos específicos del uso tecnologías de la comunicación (visuales, ergonómicos, entre otros) y alteraciones mentales debido a conflictos entre emociones "requeridas" en su trabajo y las que el trabajador quiere expresar. (ISL, 2020).

Por otro lado, dentro de los riesgos para la salud física, el sedentarismo es uno de los factores que puede favorecer o agravar el riesgo de enfermedades cardiovasculares o metabólicas, así como como el deterioro del quehacer diario o el disfrutar de vivencias diarias (ISL, 2020).

También puede desarrollar riesgos ergonómicos que pueden desencadenar sensación de fatiga, cefaleas, diarreas, palpitaciones, trastornos del sueño, tensión y dolor muscular, agotamiento, fatiga, sudoración excesiva, problemas respiratorios, mareos o vértigos, etc. (ISL, 2020)

El Tecnoestrés, puede provocar "disminución del rendimiento, problemas de sueño, insomnio, dolores de cabeza y musculares, trastornos gastrointestinales, dolor crónico, puesta en marcha de comportamientos perjudiciales como fumar, comer y beber en exceso, abuso de fármacos y otras sustancias" (ISL,2020, P3).

Finalmente, uno de los más preocupantes son los trastornos músculo esqueléticos, que se refieren a cualquier trastorno de las articulaciones o tejidos. Esto problemas pueden ir desde dolores y molestias leves hasta enfermedades crónicas que pueden producir discapacidad, e incluso impedir que el trabajador puede seguir desempeñando su labor (EU-OSHA, 2019). 


\section{c. Análisis epidemiológico relacionado al Teletrabajo y la Salud Ocupacional}

Primero se debe definir la enfermedad laboral o profesional, la que se entiende por aquella causada de manera directa por el ejercicio de la profesión que realiza una persona y que puede llegar a producir incapacidad o muerte. $Y$ debe existir una relación causal entre el quehacer laboral y la enfermedad que produzca invalidez o la muerte. (Instituto de Seguridad Social, Ministerio del Trabajo y Previsión Social, 2017).

Y el teletrabajo se entiende como, el trabajo a distancia mediante el uso de medio tecnológicos, informáticos o de telecomunicaciones o si tales servicios los debe reportar por esos medios. (Código del Trabajo, 2019)

Si bien el teletrabajo se introdujo hace varias décadas, recién en los años 90, toma real fuerza, esto por el aumento exponencial de las tecnologías de la información y la comunicación. Esto en el momento de inicio de la pandemia facilitó convertir rápidamente el trabajo presencial al remoto, un método antes opcional en esos momentos se torna como necesario. (Suasnavas R.,2021, p.2)

En virtud de la pandemia declarada en enero del 2020 por la OMS (Organización Mundial de la Salud) por COVID -19, donde el aislamiento social de la población fue uno de los pilares para prevenir la propagación de los contagios, ha sido uno de los mayores retos de estos tiempos para el área laboral, afectando a nivel económico como social. Por lo que las empresas tuvieron que resguardar la seguridad de su entidad y la conservación de los puestos de trabajo, optando por el teletrabajo o “Trabajo a distancia”. (García - Salirrosa E. 2020. p. 2)

Según la Academia Internacional de Teletrabajo el 2017, en Chile se inició el teletrabajo entre los años 2017-2018 en el Instituto Nacional de Propiedad Intelectual con el 10\% de los trabajadores. (Ramírez Velásquez, J.,2021, p.3)

Cuando se habla de teletrabajo, no solo se habla de modificar el área del hogar destinada a dicho fin, con la estructura recomendada, sino que, se adiciona la adaptación de los horarios, las medidas que se deben tener en el hogar con respecto a la seguridad y la salud, muchas veces ignoradas por los trabajadores, además de contar con un cambio en el aspecto jurídico que tiene que incluir el anexo de contrato asociado a esta modalidad de trabajo. Y esto sin considerar que, si el trabajador ignora estas medidas, puede ejecutar el teletrabajo con las condiciones ergonómicas inadecuadas y sin control en su jornada laboral. (Ramírez Velásquez, J.,2021, p.4) 
En una revisión los factores que impactaban más en la carga mundial de enfermedades profesionales, que el 2018 fue de $2.7 \%$ considerando muertes y discapacidades, se encuentra el riesgo de lesiones, exposición a gases, humos, material particulado y ruido. Por lo que la OMS y la OIT (Organización Internacional del Trabajo), desarrollaron estimaciones donde se dejó ver que los TME (trastornos musculo - esqueléticos) están asociados a cargas atribuibles a problemas ergonómicos (Baca - Cajas, K., 2021, p. 4-5).

Las disciplinas que han tomado gran relevancia en este tiempo de pandemia han sido la psicosociología o psicología social, que abarca aquellos aspectos que tiene que ver con la conducta interpersonal, en donde se revisan la totalidad de las organizaciones y su impacto en la salud mental y física de los trabajadores. Tomando como centro que en el ambiente laboral nacen los riesgos hacia la persona, y puede afectar a cualquier trabajador independiente de su edad, el tipo de riesgo, la magnitud y la frecuencia (Baca - Cajas, K., 2021, p.6).

Y, por otro lado, está la ergonomía que considera factores físicos, cognitivos, organización social y ambiente, todos en conjunto, por lo que tienen una mirada holística de los factores involucrados. Donde también se ha incorporado la carga mental como factor de riesgo ergonómico asociado a las exigencias laborales. Se intenta que la psicosociología y la ergonomía trabajen alineadas para tener una correcta adaptación de la persona al trabajo en este caso al teletrabajo (Baca Cajas, K., 2021, p. 6-7).

Si se revisa el escenario local, según el informe de accidentabilidad del año 2019, se ve un aumento en las enfermedades asociadas a trastornos musculo - esqueléticos (TME) con un 43\%, mientras que un 38\% están asociadas a los trastornos de salud mental. Finalmente, la superintendencia de Seguro Social de Chile el año 2020, indica que existe una relación de los factores psicosociales que repercuten en la salud mental y que incide en síntomas asociados a TME (Baca - Cajas, K., 2021, p.89).

De las patologías más frecuentes asociadas a los teletrabajadores están las asociadas a los TME, con la repercusión en la productividad de las empresas u organizaciones, aumentando la discapacidad por enfermedad laboral dentro de la institución. Dentro de las causas está el sobre esfuerzo, considerado un problema a nivel mundial, y la principal causa de trabajadores de oficina. Si bien varios aspectos pueden incidir en la aparición de estos trastornos, no solo físicos, si no también psicosociales y organizativos. Las problemáticas mayormente detectadas tienen que ver con los tiempos prolongados expuestos a pantalla, a movimientos repetitivos que serían los causales de los trastornos musculo - esqueléticos. Para 
lo cual, las instituciones especializadas recomiendan el abordaje desde diseñar adecuadamente la estación de trabajo considerando aspectos desde la silla hasta el tipo de pantalla. Se ha visto una relación entre el número de horas de exposición a pantalla versus el riesgo de TME, generando un impacto en los trabajadores desde trastornos de sueño, fatiga, depresión y disminución de actividades recreativas (Simbaña S, 2021, p.3).

A lo anterior se suma que es una actividad laboral completamente sedentaria, y es precisamente en este escenario que puede traer complicaciones de la índole ergonómico sobre todo alteraciones musculoesqueléticas de la y los trabajadores como los señalados anteriormente (García - Salirrosa E. 2020. p. 2).

Dentro de los factores que llevan a estas complicaciones están los factores ergonómicos, como es la postura inadecuada y mantenida, posiciones inadecuadas e incomodas, a lo que se puede sumar los horarios de trabajo, la complejidad de la labor y el entorno donde se desarrolla el teletrabajo. Según la Organización Internacional del Trabajo reportó que el año 2013 cerca de un 59\% sufrió TME (trastornos musculo esqueléticos) del total de enfermedades profesionales. el año 2017 fue la 2da causa a nivel mundial de invalidez(García - Salirrosa E. 2020. p. 2).

El costo económico que significa para los distintos países es alto, por ejemplo, en la Unión Europea el gasto asociado a enfermedades y accidente de trabajo osciló entre 2.6 y $3.8 \%$ del PIB (producto interno bruto). En EE.UU. los días perdidos por discapacidad tienen un costo cercano a los 215 millones de dólares al año, y en américa latina significan de un 9 al 12\% del PIB (García - Salirrosa E. 2020. p. 2).

En un estudio en docentes de universidades públicas y privadas de Lima, Perú, que estaban en modalidad de teletrabajo, dentro de los resultados obtenidos, el rango etario predominante fue de 41 a 50 años, mayor en sexo masculino, con promedio de 8 a 10 y más horas frente a la pantalla, de un total de 110 docentes 91 estaban conectados de 5 a 7 días a la semana. Dentro de los síntomas más frecuentes se encontraron el malestar a nivel de cuello, hombro y región dorso - lumbar (García - Salirrosa E. 2020. p. 3-6).

Si bien la Comisión Económica para América Latina, vio en el teletrabajo, la oportunidad de resguardar los puestos de trabajo en el contexto de COVID - 19, asegurando continuidad en la producción de distintas áreas, con lo que disminuía el riesgo de exposición al virus, con los cambios legales y administrativos que se tuvieron que adoptar, pero la revista médica "The Lancet", después de estudios desarrollados en virtud del impacto del confinamiento, en los resultados encontraron que habían desarrollado depresión, estrés, mal humor, irritabilidad, 
insomnio, síntoma de estrés postraumático, ira y agotamiento, esto se vio asociado al aislamiento que tuvieron que tener por el confinamiento (Baca - Cajas, K., 2021, p. 9).

\section{d. Contexto del teletrabajo y conciliación con la vida familiar}

Al aplicar el Teletrabajo se pueden presentar ciertas dificultades que van relacionadas con la privacidad del trabajador ya que se puede entrar en conflicto al utilizar el hogar como centro de trabajo (Duran, 2020).

El Teletrabajo para muchas Organizaciones no fue una opción sino una necesidad para continuar las actividades laborales, económicas en respuesta a la Emergencia sanitaria. (Duran, 2020) Por lo lado "los estudios han demostrado que el teletrabajo aumenta la intensidad del trabajo y la interferencia entre el trabajo y el hogar, lo que provoca efectos adversos en el bienestar y los niveles de estrés de los teletrabajadores" (OIT, 2020, p.18).

Por otra parte, el Teletrabajo implica el encuentro de diferentes mundos, donde múltiples factores individuales, familiares, organizativos, contextuales y socioculturales llevan a adaptaciones y significaciones también particulares (Duran, 2020, Ordoñez, 2012).

Tomando en cuenta que el Teletrabajo se desarrolla en el ámbito de convivencia familiar, es importante involucrar a la familia o las personas con quien uno convive en el cambio de modalidad de trabajo, porque teletrabajar significa un cambio no solo para la y el trabajador, sino para su familia o para las personas con quienes convive (Duran, 2020).

La combinación del Teletrabajo con la convivencia familiar no siempre es compacto, lineal, homogéneo, puede presentarse tensiones, paradojas, sin embargo, es válido reconocer si se establece ciertas acciones que promueven el equilibrio de ambas, se percibirá los beneficios que aporta el rendimiento en el Teletrabajo (Duran, 2020).

La clave está en involucrar a la familia y personas con quien vive para que sean un apoyo en el nuevo proceso y faciliten la convivencia durante la jornada laboral, es importante definir los tiempos laborales y respetar los espacios en donde la y el teletrabajador estará, es muy importante explicarles las reglas de juego a los integrantes de la familia, llegar a acuerdos y compartirles los beneficios de implementar esta modalidad (Duran, 2020).

Para conciliar este conflicto entre el trabajo y la convivencia con hijos en el hogar, existen múltiples recomendaciones básicas, tales como: Organizar su tiempo y acuerda con el empleador un trabajo por metas, y condiciones básicas de salud y 
seguridad en el trabajo para su hogar Por otro lado, se dictan recomendaciones básicas para aplicar con los hijos/as, entre estas se encuentran: tomar tiempo para jugar con ellos; en el caso de menores de un año, se sugiere establecer estaciones dentro de la casa para que el niño o niña realice actividades diferenciadas; en niños en edad escolar se recomienda crear un calendario y ofrecer opciones de juego, estudio y aventura; y en adolescentes mantener una estructura de estudio, juego, desafíos y tareas hogareñas, y a través de ellas establezca objetivos que puedan ser evaluados y fomentados por toda la familia. Necesarias son paciencia, comunicación y el amor filial (Lizondo, Silva, Arancibia, Cortés \& Muñoz, 2021).

Tabla $N^{\circ} 1$ Resumen del análisis de la implementación del teletrabajo.

$\checkmark$ En Chile la Ley 21.220, plantea que el trabajador presta sus servicios, desde su domicilio u otro lugar distinto a las instalaciones de la empresa (Ley 21220, 2020).

$\checkmark$ El teletrabajo conlleva un trabajo realizado con la ayuda de las TIC, fuera de las instalaciones del empleador (OIT, 2020).

$\checkmark \quad$ El empleador debe respetar el derecho a desconexión (Ley 21220, 2020).

Legislación Laboral $\quad \checkmark$ Son responsabilidad del empleador la entrega de referente al Teletrabajo. equipos, herramientas y los materiales, incluyendo elementos de protección personal (Ley 21220, p2).

$\checkmark$ Los trabajadores que tengan modalidad teletrabajo tendrán iguales derechos y obligaciones en materia de seguridad y salud en el trabajo que cualquier otro trabajador (Decreto 18, 2020, p1).

$\checkmark$ Quedando sujetos a todas las materias por la exposición a riesgos a causa o con ocasión del trabajo (Ley 16.744, 2017)

$\checkmark$ El teletrabajo provoca generación de estrés, ansiedad, irritabilidad, estados depresivos, temor y aburrimiento, sensación de aislamiento, ambigüedad o conflicto de rol, (ISL, 2020).

$\checkmark$ En la salud física, el sedentarismo aumenta el riesgo de enfermedades cardiovasculares (ISL, 2020).

Riesgos para la Salud del desencadenar Sensación de fatiga, cefaleas, diarreas Teletrabajo. palpitaciones, agotamiento, fatiga, (ISL, 2020)

$\checkmark$ El Tecnoestrés, puede provocar problemas de sueño, insomnio, dolores de cabeza, trastornos gastrointestinales, puesta en marcha de comportamientos perjudiciales como fumar, comer y beber en exceso (ISL,2020).

$\checkmark$ Desarrollo de trastornos músculo esqueléticos, a través de dolores, molestias leves, enfermedades crónicas y discapacidad,(EU-OSHA, 2019).

Análisis epidemiológico $\quad \checkmark$ La enfermedad laboral o profesional, se entiende por relacionado al Teletrabajo aquella causada de manera directa por el ejercicio de la 
y la Salud Ocupacional.

\section{Contexto del teletrabajo y conciliación con la vida familiar.}

profesión que realiza una persona (Instituto de Seguridad Social, Ministerio del Trabajo y Previsión Social, 2017).

$\checkmark$ EL teletrabajo se entiende como, el trabajo a distancia mediante el uso de medio tecnológicos, informáticos o de telecomunicaciones (Código del Trabajo, 2019)

$\checkmark$ EL teletrabajo, requiere modificar el área del hogar destinada a dicho fin, con una estructura recomendada, y la adaptación de horarios, pero estas medidas de seguridad y la salud, son ignoradas por los trabajadores. (Ramírez Velásquez, J.,2021, p.4)

$\checkmark$ La psicosociología o psicología social, tomo fuerza como disciplina en la pandemia, por los diversos problemas que se desarrollaron por el aislamiento y el teletrabajo. (Baca - Cajas, K., 2021, p.6)

$\checkmark$ Hay relación entre el número de horas de exposición a pantalla versus el riesgo de TME, generando un impacto en los trabajadores desde trastornos de sueño, fatiga, depresión y disminución de actividades recreativas. (Simbaña S, 2021, p.3)

$\checkmark$ Los factores que llevan a estas complicaciones son la postura inadecuada y mantenida, posiciones inadecuadas e incomodas, a lo que se puede sumar los horarios de trabajo, la complejidad de la labor y el entorno donde se desarrolla el teletrabajo. (García Salirrosa E. 2020. p. 2)

$\checkmark$ El costo económico que significa para los distintos países es alto, en la Unión Europea el gasto asociado a enfermedades y accidente de trabajo osciló entre 2.6 y $3.8 \%$ del PIB (producto interno bruto). En EE.UU. los días perdidos por discapacidad tienen un costo de 215 millones de dólares al año, y en américa latina significan de un 9 al 12\% del PIB. (García - Salirrosa E. 2020.)

$\checkmark$ El teletrabajo fue la oportunidad de resguardar los puestos de trabajo en el contexto de COVID - 19, pero desencadeno en los teletrabajadores depresión, estrés, mal humor, irritabilidad, insomnio, síntoma de estrés postraumático, ira y agotamiento, por el confinamiento. (Baca - Cajas, K., 2021, p. 9)

$\checkmark$ Existe problemas de privacidad por utilizar el hogar como centro de trabajo. (Duran, 2020)

$\checkmark$ El Teletrabajo fue una necesidad para continuar las actividades debido a la Emergencia sanitaria. (Duran, 2020)

$\checkmark$ El teletrabajo provoca un choque entre el trabajo y el hogar, aumentando los niveles de stress (OIT, 2020).

$\checkmark$ Se debe involucrar a la familia en el cambio de modalidad de trabajo (Duran, 2020)

$\checkmark$ Se deben definir tiempos laborales y respetar los espacios en donde se teletrabajador (Duran, 2020) 


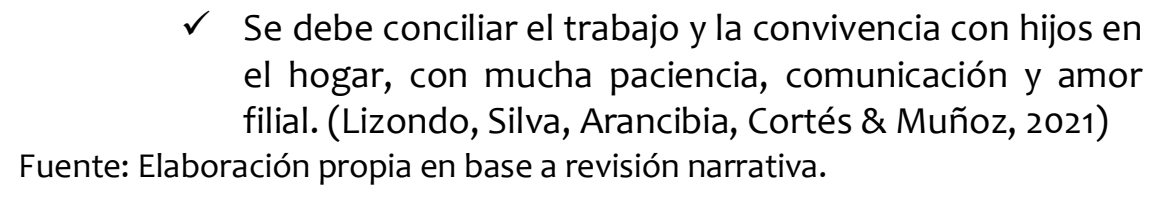

\section{Recomendaciones para el Teletrabajo:}

La Organización mundial del trabajo (OIT), en su guía práctica para el teletrabajo recomienda considerar una seria de acciones para adaptar el teletrabajo en tiempos de pandemia, afín de que la implementación del teletrabajo garantice el bienestar de sus trabajadores y permita mantener la continuidad de la producción de bienes y servicios. (OIT, 2020).

El desarrollo de la pandemia de COVID19 ha generado la introducción de una serie de medidas restrictivas como el distanciamiento físico, los cierres y las restricciones conexas para aplanar la curva de infecciones la que ha impactado enormemente el mundo laboral, generando entre otras, "licencias (obligatorias) de duración incierta, licencias sin sueldo, reducción de las horas de trabajo y los salarios, despidos y pérdida de puestos de trabajo, así como cierres de empresas" (OIT, 2020, p5). Sin embargo, un importante segmento de la población activa laboralmente ha tenido un incremento de la carga laboral y cambios en las condiciones de trabajo.

Tabla N 2: Recomendaciones para el Teletrabajo:

Tiempo de trabajo y

organización del trabajo

Gestión del desempeño

Digitalización

Comunicación

Seguridad y salud en

el trabajo (SST)

Consecuencias

jurídicas y

contractuales

Formación
Gestiona la organización de su tiempo de trabajo, priorizando adecuadamente los horarios de trabajo y los dedicados a la familia y actividades de ocio.

Ser muy claro sobre los resultados esperados, Brindando retroalimentación positiva, oportuna, regular y descriptiva de lo esperado.

Revisar las necesidades de tecnología y los recursos de los empleados que trabajan desde casa y disponer por parte del empleador de estas, o compensar en caso necesario.

Establecer normas de comunicación, resguardo de datos y seguridad de estos.

Aplicar normativa de SST, con iguales derechos y obligaciones que cualquier trabajador, incluyendo el apoyo y acompañamiento. Información pertinente respecto a condiciones contractuales y consecuencias jurídicas, de acuerdo con la legislación vigente, por parte del empleador.

Realizar diagnósticos sobre necesidades de capacitación de carácter participativo y de acuerdo con necesidades detectadas, desarrollar planes de formación que no sobrecarguen al Revista Pensamiento Académico de la Universidad UNIACC Vol. $4 \mathrm{~N}^{\circ} 1,2021$ 
trabajador.
El equilibrio entre el trabajo y la vida privada

Dimensión de género del teletrabajo
Gestionar los límites entre la vida laboral y la vida privada estableciendo expectativas claras sobre los resultados del trabajo y respecto de la flexibilidad de gestionar sus propios horarios de trabajo en función de sus necesidades individuales.

Comprender cómo distintos factores afectan de manera diferente a los hombres y a las mujeres.

Fuente: Adaptado de la "El teletrabajo durante la pandemia de COVID-19 y después de ella - Guía práctica" (OIT, 2020).

\section{Conclusión}

En base a revisión narrativa realizada se encontraron cuatro categorías emergentes, la Legislación Laboral referente al Teletrabajo, los Riesgos para la Salud del Teletrabajo, Análisis epidemiológico relacionado al Teletrabajo y la Salud Ocupacional, y el Contexto del teletrabajo y conciliación con la vida familiar. A partir de estos ejes se describen una serie de recomendaciones para mejorar la implementación del teletrabajo en condiciones de Pandemia.

En relación con la Legislación Laboral referente al Teletrabajo, se encontró que Chile no cumple con los requisitos de UE presentando un vacío legal que va en desmedro del teletrabajador, en la que no se incorporan las medidas de seguridad mínimas para este, ni siquiera en forma de capacitación o indicaciones, ni tampoco la entrega de los elementos de trabajo para el hogar o los costos asociados a estos.

En la lógica de los Riesgos para la Salud del Teletrabajo, el teletrabajo puede provocar generación de estrés, ansiedad, irritabilidad, estados depresivos, temor y aburrimiento, sensación de aislamiento, ambigüedad o conflicto de rol, aumentar el sedentarismo y por consíguete el riesgo de enfermedades cardiovasculares, desde la ergonómia se pueden desencadenar sensación de fatiga, cefaleas, diarreas palpitaciones, agotamiento, fatiga, así mismo el Tecnoestrés, puede provocar problemas de sueño, insomnio, dolores de cabeza, trastornos gastrointestinales, puesta en marcha de comportamientos perjudiciales como fumar, comer y beber en exceso.

En la categoría del Análisis epidemiológico relacionado al Teletrabajo y la Salud Ocupacional, se puede encontrar evidencia de que el teletrabajo puede incrementar riesgos laborales relacionados a los trastornos musculo esqueléticos, problemas de salud mental, así como agudizar factores de riesgo de la salud de las personas como el sedentarismo, la obesidad, problemas con los órganos sensoriales, entre otros. 
Por otro lado, existen una serie de factores que no son evaluados en condiciones de teletrabajo, y que dicen relación con las condiciones propias del hogar, como el ruido, exposiciones a gases, agentes biológicos entre otros, que son de difícil identificación y control. Por lo anterior muchos de los teletrabajadores podrían presentar enfermedades o accidentes que podrían enmascararse con condiciones de salud común y con ello incrementar la subnotificación de enfermedades o accidentes que se produzcan durante el periodo que dure el teletrabajo.

En la dinámica de la relación familiar, la bibliografía consultada revela que persisten desigualdades relaciones al género, siendo las mujeres las más perjudicadas y con una relación desbalanceada entre teletrabajo y roles familiares, entregándoles una carga mayor a la de los varones, esto debido a que los niños y niñas no están en el colegio de menara presencial, sino que también en modalidad telemática, lo que dificultaba la situación laboral-familiar.

Existen recomendaciones para prevenir problemáticas de salud mental y física que pueden y deben ser implementadas por los estados en todo el mundo, siguiendo lineamientos de los organismos internacionales especializados, relevando las disciplinas de ergonomía y psicología laboral, entre otras resumidas en la figura $\mathrm{N}^{\circ} 1$, para abordar condiciones laborales en teletrabajo, que es muy probable, se mantenga durante un largo periodo de tiempo y que puedan repetirse en el futuro.

En base al análisis de las categorías identificadas, podemos decir que la implementación del teletrabajo se realizado en un modo de emergencia debido al COVID-19 y bajo una legislación insuficiente para proteger la salud de teletrabajadores, existiendo enormes vacíos en las áreas de formación e implementación de medidas de seguridad en el lugar de trabajo del teletrabajador. Esto puede incrementar el riego de sufrir trastornos de salud física, mental y social, acrecentado con la situación de confinamiento. Por lo anterior es de vital importancia identificar y notificar oportunamente posibles trastornos de salud tanto en teletrabajadores y sus familias, de tal manera de evitar la subvaloración de la problemática actual y futura, las que pueden tener consecuencias insospechadas para la realidad laboral, productiva y de calidad de vida de la población trabajadora y sus familias. 


\section{Referencias}

Baca-Cajas, K. A. (2021). Evaluación ergonómica y psicosocial de puesto administrativo y su contraste al actual teletrabajo por pandemia. Revista Científica FIPCAEC (Fomento De La investigación $Y$ publicación En Ciencias Administrativas, Económicas Y Contables). Polo De Capacitación, Investigación $Y$ Publicación (POCAIP), 6(3), 327-366. https://doi.org/10.23857/fipcaec.v6i3.402

Bonilla, L., Plaza, D., De Cerquera, G. y Riaño-Casallas, M. (2014). Teletrabajo y su Relación con la Seguridad y Salud en el Trabajo. Ciencia \& trabajo, 16(49), 3842. https://dx.doi.org/10.4067/S0718-24492014000100007

Buitrago, D. (2020). Teletrabajo: una oportunidad en tiempos de crisis. Revista CES Derecho, 11(1), 1-2. http://www.scielo.org.co/scielo.php?script=sci_arttext\&pid=S214577192020000100001\&lng=en\&tlng=es

Código del Trabajo Artículo 152 https://www.dt.gob.cl/portal/1626/articles95516 recurso_2.pdf

Codina, L. (2020). Cómo hacer revisiones bibliográficas tradicionales o sistemáticas utilizando bases de datos académicas. Revista ORL, 11(2), 139-153. https://dx.doi.org/10.14201/orl.22977

Decreto 18 de 2020. Diario Oficial de la República de Chile, 23 de Abril de 2020. http://bcn.cl/2ep6h

Duran, N. (2020). El Teletrabajo y la conciliación con el entorno de convivencia familiar durante la Pandemia COVID-19. Revista de Investigación Psicológica, (Especial), 68-72. http://www.scielo.org.bo/scielo.php?script=sci_arttext\&pid=S222330322020000300009\&lng=es\&tlng=es.

European Agency for Safety and Health at Work (EU-OSHA) (2019). Work-related musculoskeletal disorders: prevalence, costs and demographics in the EU. European Risk Observatory. Report. https://dx.doi.org/10.2802/66947

García-Salirrosas E, Sánchez-Poma R. Prevalencia de trastornos musculoesqueléticos en docentes universitarios que realizan teletrabajo en tiempos de COVID-19. An Fac med. 2020;81(3):301-7. https://doi.org/10.15381/anales.v81i3.18841

Guirao, S. (2015). Utilidad y tipos de revisión de literatura. Ene, 9(2)https://dx.doi.org/10.4321/S1988-348X2015000200002 
Instituto de Seguridad Laboral (ISL) (2020). Riesgos Psicosociales en el Trabajo, Recomendaciones para el Autocuidado. Ministerio del Trabajo y Previsión Social. Gobierno de Chile. https://www.isl.gob.cl/wpcontent/uploads/Teletrabajo-Recomendaciones-Autocuidado.pdf

Ley 16.744 de 2017. http://bcn.cl/299zk

Ley 21.220 de 2020. http://bcn.cl/2dl8j

Lizondo-Valencia, R., Silva, D., Arancibia, D., Cortés, F., y Muñoz-Marín, D. (2021). Pandemia y niñez: Efectos en el desarrollo de niños y niñas por la pandemia Covid-19. Veritas \& Research, 3(1), 16-25.

http://revistas.pucesa.edu.ec/ojs/index.php?journal=VR\&page=article\&op=vie w\&path[]=75

López, N., Pérez-Simon, M., Nagham-Ngwessitcheu, E. y Vázquez-Ubago, M. (2014). Teletrabajo, un enfoque desde la perspectiva de la salud laboral. Medicina y Seguridad del Trabajo, 60(236), 587-599. https://dx.doi.org/10.4321/S0465546X2014000300009

Oficina Internacional del Trabajo (OIT), El teletrabajo durante la pandemia de COVID-19 y después de ella - c Guía práctica https://www.ilo.org/global/publications/WCMS_758007/lang--es/index.htm

Organización Internacional del Trabajo (OIT). (2020) El teletrabajo durante la pandemia de COVID-19 y después de ella - Guía práctica. https://www.ilo.org/wcmsp5/groups/public/---ed_protect/---protrav/--travail/documents/publication/wcms_758007.pdf

Osio, L. (2010). El Teletrabajo: Una opción en la era digital. Observatorio Laboral Revista Venezolana, 3 (5), 93-109. https://www.redalyc.org/articulo.oa?id=219014912006

Ramírez, J., Quinde, L., Alarcón, R., y Vega, C. (2021). Teletrabajo en tiempos de pandemia: Un reto laboral en la educación superior. Revista Scientific, 6(20), 130-151. https://doi.org/10.29394/Scientific.issn.2542-2987.2021.6.20.7.130-151

Rodríguez, M. (2007). El teletrabajo en el mundo y Colombia. Gaceta Laboral , 13(1), 29-42. http://ve.scielo.org/scielo.php?script=sci_arttext\&pid=S1315$85972007000100002 \& \operatorname{lng}=$ es\&tlng=es. 
Salinas, M. (2020). Sobre las revisiones sistemáticas y narrativas de la literatura en Medicina. Revista chilena de enfermedades respiratorias, 36(1), 26-32. https://dx.doi.org/10.4067/S0717-73482020000100026

Silva, J. (2014). Flexibilidad y teletrabajo: un estudio bibliométrico sobre la producción científica. Perspectivas em Ciência da Informação, 19(2), $159-173$. https://doi.org/10.1590/1981-5344/1884

Simbaña, S. (2021). Prevalencia de trastornos musculoesqueléticos por posturas forzadas en trabajadores que realizan teletrabajo en una institución financiera. (Tesis de grado magister). Universidad Internacional SEK. Quito, Ecuador.

Suasnavas, R. (2021). Identificación de riesgo en usuarios de pantallas de visualización de datos en condiciones laborales de teletrabajo de la empresa (Tesis de grado magister). Universidad Internacional SEK.

Vera, O. (2009). Cómo escribir artículos de revisión. Revista Médica La Paz, 15(1), 6369. http://www.scielo.org.bo/scielo.php?script=sci_arttext\&pid=S172689582009000100010\&lng=es\&tlng=es 


\section{Fernando Cortes Tello}

Enfermero, Universidad de Antofagasta, Magíster en Salud Pública Universidad de Chile y Magíster en Didáctica para la Educación Superior de la Universidad de Tarapacá, Auditor líder en sistemas integrados de Gestión. Director de Carrera de Enfermería de la Universidad Central de Chile, Región de Coquimbo. Investigador de Grupo de Investigación en Gestión Sanitaria (GIGES). https://orcid.org/0000-0002-0217-4608

Email: fernando.cortes@ucentral.cl

Diego Silva Jiménez

Administrador Público y Magister en Gerencia y Políticas Públicas, Universidad de Santiago de Chile, @Doctor en Educación con mención en gestión educativa, @Magíster en Ciencias de la Educación Mención Docencia e Investigación Universitaria, Universidad Central de Chile. Coordinador Área de Gestión y Metodología de la Investigación, de la Unidad de Salud Pública del Departamento en Formación Transversal en Salud, de la Facultad de Ciencias de la Salud Universidad Central de Chile. Coordinador Grupo de Investigación en Gestión Sanitaria (GIGES). https://orcid.org/0000-0003-2818-211X

Email: diego.silva@ucentral.cl

\section{Daniela Muñoz Marín}

Enfermera de la Universidad Mayor, @ Magister en Docencia para la Educación Superior, Universidad Central de Chile, Coordinadora de Campos Clínicos de la carrera de enfermería sede Coquimbo. Investigadora de Grupo de Investigación en Gestión Sanitaria (GIGES).

https://orcid.org/0000-0001-6702-0231

Email: daniela.munoz@ucentral.cl

\section{Romina Lizondo Valencia}

Enfermera, Universidad Mayor, Magíster Gestión Educacional, Universidad Andrés Bello con doble titulación en la Universidad Europea de Madrid, Magíster en Ciencias de la educación, mención Docencia e Investigación Universitaria, Universidad Central de Chile, Coordinadora de Campos clínicos de Enfermería Santiago, de la Facultad de Ciencias de la Salud Universidad Central de Chile. Investigadora de Grupo de Investigación en Gestión Sanitaria (GIGES). https://orcid.org/0000-0003-1085-9524

Email: rlizondov@ucentral.cl 